\title{
English Cross-Cultural Translation and Validation of the Neuromuscular Score: A System for Motor Function Classification in Patients With Neuromuscular Diseases
}

\author{
Carole Vuillerot, MD, PhD ${ }^{a, b, c, d}$, Katherine G. Meilleur, PhD ${ }^{\mathrm{e}}$, Minal Jain, PT, DSc ${ }^{\mathfrak{f}}$, Melissa $^{2}$ \\ Waite, PT $^{f}$, Tianxia Wu, PhDa ${ }^{a}$, Melody Linton, BS ${ }^{e}$, Jahannaz Datsgir, DOa ${ }^{a}$, Sandra \\ Donkervoort, MS, CGCa, Meganne E. Leach, MSN, PNP-BCa,g, Anne Rutkowski, MD ${ }^{\mathrm{h}}$, \\ Pascal Rippert, PhD $^{\mathrm{b}, \mathrm{i}}$, Christine Payan, MDj, Jean Iwaz, PhD ${ }^{\mathrm{c}, \mathrm{d}, \mathrm{k}}$, Dalil Hamroun, MD', \\ Carole Bérard, $\mathbf{M D}^{\mathrm{b}}$, Isabelle Poirot, $\mathbf{M D}, \mathbf{P h D}^{\mathrm{b}}$, and Carsten G. Bönnemann, $\mathbf{M D}^{\mathrm{a}}$ \\ aNeuromuscular and Neurogenetic Disorders of Childhood Section, National Institute of \\ Neurological Disorders and Stroke, National Institutes of Health, Bethesda, MD \\ 'bospices Civils de Lyon, L'Escale, Pediatric Physical Medicine and Rehabilitation Department, \\ Bron, France \\ 'Université de Lyon, Lyon, France

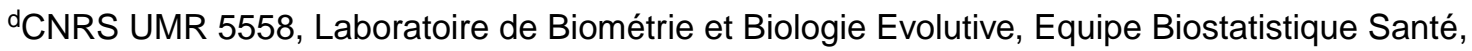 \\ Pierre-Bénite, France \\ eNational Institute of Nursing Research, National Institutes of Health, Bethesda, MD \\ ${ }^{f}$ Mark O. Hatfield Clinical Research Center, National Institutes of Health, Bethesda, MD \\ gChildren's National Health System, Washington. DC \\ ${ }^{\text {h}}$ Cure Congenital Muscular Dystrophy and Kaiser Southern California Permanente Medical \\ Group, Los Angeles, CA \\ 'Hospices Civils de Lyon, Pôle Information Médicale Évaluation Recherche, Lyon, France \\ jAssistance Publique-Hôpitaux de Paris, Hôpital Pitié-Salpétrière, Department of Clinical \\ Pharmacology, Paris, France \\ kHospices Civils de Lyon, Service de Biostatistique, Lyon, France \\ 'Centre Hospitalo-Universitaire de Montpellier, Montpellier, France
}

\section{Abstract}

Corresponding authors. Carole Vuillerot, MD, PhD, Service L'Escale, Hôpital Femme Mère Enfant, 59 Boulevard Pinel, F-69677 Bron Cedex, France. carole.vuillerot@ chuyon.fr., Carsten G. Bonnemann, MD, Neuromuscular and Neurogenetic Disorders of Childhood Section, National Institute of Neurological Disorders and Stroke, National Institutes of Health, 35 Convent Dr, Bldg 35 , Rm 2A-116, Bethesda, MD 20892-3705. carsten.bonnemann@nih.gov.

Disclosures: none. 
Objective-To develop and validate an English version of the Neuromuscular (NM)-Score, a classification for patients with NM diseases in each of the 3 motor function domains: D1, standing and transfers; D2, axial and proximal motor function; and D3, distal motor function.

Design-Validation survey.

Setting-Patients seen at a medical research center between June and September 2013.

Participants-Consecutive patients $(\mathrm{N}=42)$ aged 5 to 19 years with a confirmed or suspected diagnosis of congenital muscular dystrophy.

Interventions-Not applicable.

Main Outcome Measures-An English version of the NM-Score was developed by a 9-person expert panel that assessed its content validity and semantic equivalence. Its concurrent validity was tested against criterion standards (Brooke Scale, Motor Function Measure [MFM], activity limitations for patients with upper and/or lower limb impairments [ACTIVLIM], Jebsen Test, and myometry measurements). Informant agreement between patient/caregiver (P/C)-reported and medical doctor (MD)-reported NM scores was measured by weighted kappa.

Results-Significant correlation coefficients were found between NM scores and criterion standards. The highest correlations were found between NM-score D1 and MFM score D1 ( $\rho=-$. $944, P<.0001)$, ACTIVLIM ( $\rho=-.895, P<.0001)$, and hip abduction strength by myometry $(\rho=$ $-.811, P<.0001)$. Informant agreement between P/C-reported and MD-reported NM scores was high for D1 $(\kappa=.801 ; 95 \%$ confidence interval $[\mathrm{CI}], .701-.914)$ but moderate for D2 $(\kappa=.592$; 95\% CI, .412-.773) and D3 ( $\kappa=.485 ; 95 \%$ CI, .290-.680). Correlation coefficients between the $\mathrm{NM}$ scores and the criterion standards did not significantly differ between P/C-reported and MDreported NM scores.

Conclusions-Patients and physicians completed the English NM-Score easily and accurately. The English version is a reliable and valid instrument that can be used in clinical practice and research to describe the functional abilities of patients with NM diseases.

\section{Keywords}

Activities of daily living; Disability evaluation; Neuromuscular diseases; Rehabilitation

According to Rossi et al, ${ }^{1}$ neuromuscular diseases (NMDs) encompass "all disorders caused by an abnormality of any component of the lower motor neuron system: anterior horn cell, peripheral nerve, neuromuscular junction, or muscle." In fact, whatever the cause, most NMDs involve impairment and most frequently a progressive decline of motor function.

There is an increasing interest in developing valid outcome measures to assess motor function and describe the effects of interventions especially in multinational trials ${ }^{2-4}$; thus, several functional quantitative scales have been developed. Some are disease-specific (eg, Hammersmith Motor Ability Score, ${ }^{5}$ Modified Hammersmith Functional Motor Scale, ${ }^{6}$ expanded version of the Hammersmith Functional Motor Scale, ${ }^{4}$ North Star Ambulatory Assessment ${ }^{7}$ ); others, such as the Motor Function Measure (MFM), ${ }^{8}$ are designed for all NMDs and applicable regardless of the patient's ambulant status. These quantitative scales measure motor functional capabilities under controlled conditions and are administered by 
trained physical therapists within 20 to 30 minutes. Nevertheless, several scales lack the sufficient sensitivity to change that would enable detecting the effects of interventions on motor function in clinical trials.

The work by Palisano's team ${ }^{9}$ (ie, the Gross Motor Function Classification System [GMFCS] in cerebral palsy) emphasizes clinical severity scales/classifications that describe a patient's functional status. These classification systems are helpful in clinical research to stratify patients of equal abilities, thereby reducing sample variability. Previous functional rating scales developed specifically for individuals with dystrophinopathies did not consider all components of motor function; Brooke $\mathrm{e}^{10,11}$ and Vignos ${ }^{12}$ and colleagues grades describe only the upper body function and the ambulatory capacities, respectively.

To address the need for a standardized method to classify the motor functions of patients with NMDs, the Neuromuscular (NM)-Score was first developed and validated in French. ${ }^{13}$ Based on a statement by the World Health Organization about the potential influence of personal and environmental factors on the way patients with NMDs manifest their mobility capacities, ${ }^{9}$ the NM-Score focuses on motor function performance under typical current circumstances, not under controlled test conditions.

To extend the applicability of the NM-Score to English-speaking countries, the NM-Score was translated and cross-culturally adapted and is here tested for concurrent validity against other established outcome measurements (MFM, ${ }^{8}$ ACTIVLIM [activity limitations for patients with upper and/or lower limb impairments $],{ }^{14}$ Brooke Scale, ${ }^{10,11}$ Jebsen-Taylor Hand Function Test, ${ }^{15}$ and selected myometry measurements) in a cohort of patients with congenital muscular dystrophy (CMD). Because this instrument can be equally used by both patients and parents (the most familiar with the patient's performance in daily living), informant agreement between patient-reported and clinician-reported clinical severity scores was also assessed.

\section{Methods}

This study is part of a National Institutes of Health (NIH) protocol entitled "Clinical and Molecular Manifestations of Neuromuscular and Neurogenetic Disorders of Childhood" (Clinical Trial Registration No.: NCT01568658) and has obtained the ethical approval from the NIH Institutional Review Board. Participant consents were obtained according to the Declaration of Helsinki.

\section{NM-Score classification}

The NM-Score is a questionnaire-based clinical severity score first developed in French to assess the functional ability of patients with NMDs in 3 domains: standing position and transfers (D1), axial and proximal motor function (D2), and distal motor function (D3).

A rigorous methodology of development and validation of the NM-Score has demonstrated its content validity through a Delphi process that involved 23 experts. ${ }^{13}$ Its reproducibility was considered good. For D1, D2, and D3, the levels of agreement between raters were $82.9 \%, 78.1 \%$, and $75.6 \%$, and the kappa coefficients were .77, .69, and .64, respectively. 
The disagreements were minimal, a maximum of 1 point whatever the domain. There were significant correlations between the NM scores and the MFM scores: -.881 for D1, -.702 for D2, and -.495 for D3. In addition, the correlations were strong between the NM score D2 and Brooke Scale $(r=.642)$, and between the NM score D1 and Vignos Scale $(r=.862)$.

\section{Translation and adaptation of NM-Score into English}

Guillemin et al ${ }^{16}$ proposed a set of standardized guidelines for cross-cultural adaptation of health-related quality-of-life measures based on previous research in psychology, sociology, and published methodological frameworks. These guidelines include recommendations for obtaining semantic, idiomatic, experiential, and conceptual equivalence in translation by using back-translation techniques and committee reviews.

In accordance with these guidelines as described in figure 1, two independent French to English translations were completed and compared, thereby leading to a reference version. The cultural sensitivity of the questionnaire phraseology was carefully considered because of the difference in the approach to disability between the French and the American culture. Then, a back-translation of the reference version was completed and compared with the initial French version by a committee of NMD experts (physical therapists, physicians, nurse practitioners) to identify any misinterpretations and finalize the NM-Score English version (appendix 1).

\section{Validation study of English NM-Score}

As per the NIH protocol, all the participants were diagnosed with CMD (clinical, muscle biopsy \pm genetic testing): collagen 6-related disease, laminin alpha 2-related disease (LAMA2-RD), or unclassified CMD. Their functional abilities were assessed by skilled physical therapists using standardized assessment tools including MFM, ACTIVLIM, Brooke Scale, Jebsen-Taylor Hand Function Test, and myometry.

The MFM measures functional abilities in persons with NMDs who are 2 to 60 years of age. ${ }^{8,13}$ The ACTIVLIM is a self-administered 20 -item questionnaire that measures activity limitation in patients with upper or lower limb impairments, or both. ${ }^{14}$ The Brooke Scale is a 6-point ordinal scale that assesses the upper limb ability. ${ }^{10,11}$ The Jebsen-Taylor Hand Function Test is a standardized and objective evaluation of hand function through simulated activities of daily living. ${ }^{15}$ Myometry measurements (hip abduction, knee extension, elbow extension) were carried out according to a standardized method. Myometry data were expressed as percentages of the reference values based on sex, age, and weight. ${ }^{17}$

In each patient, the NM-Score was rated independently by a physician (medical doctor [MD]) (MD_NM-Score) and by the patient or caregiver, or by both (patient/caregiver [P/C]) (P/C_NM-Score). The P/C version included a descriptive paragraph about the study and a request to provide feedback about its usefulness and limitations. The processes of questionnaire administration and scoring of this $\mathrm{P} / \mathrm{C}$ version were standardized. 


\section{Statistical analysis}

Quantitative variables were described as means, SDs, and ranges. Categorical variables were described as frequencies or percentages. A 2-way analysis of variance was used to compare the MD_NM-Scores between the 3 diagnosis groups (collagen 6-related disease, LAMA2$\mathrm{RD}$, and unclassified CMD) and the 2 ambulant status groups (ambulant and nonambulant). The product term for interaction between diagnosis and ambulant status was considered but finally dropped from the model ( $P>0.2)$. A Box-Cox transformation was applied to the NM scores and to other variables that did not follow normal distributions.

Spearman correlation coefficients were used to evaluate the relationship between the 2 sets of measurements: (1) NM scores D1, D2, and D3 (MD and P/C) and (2) the standard instruments (MFM, ACTIVLIM, Brooke Scale, Jebsen-Taylor Test, and myometry). A $P$ value of .01 was used as the significance level to adjust for multiple tests.

The strength of agreement between P/C_NM-Scores and MD_NM-Scores was assessed by Cohen's weighted kappa coefficient $(\kappa)$, a measure of chance-corrected agreement. This kappa was interpreted as poor $(<0.4)$, moderate $(0.4-.59)$, good $(0.6-0.8)$, or excellent $(>0.8) .{ }^{18}$

\section{Results}

Overall, 42 patients ( 23 boys, 19 girls) aged 5 to 19 years (mean age \pm SD, $10.8 \pm 3.5 \mathrm{y}$ ) were enrolled in the current validation study. All MD_NM-Scores were obtained after patient observation, examination of medical records, and interviews with the patients or their caregivers. Most of the P/C_NM-Scores (27) were obtained from patients and their caregiver together, where as only 2 were obtained from the patient alone and 13 from the caregiver alone. The feedback provided by the patients and the caregivers was positive. Only 3 families expressed the need for clear definitions of some medical terms.

Table 1 describes the patients' characteristics with the medians and the ranges of the MD_NM-Scores and P/C_NM-Scores in the 3 domains D1, D2, and D3 according to the 3 diagnosis groups. As expected from the disease phenotypes, D1 was the most severely affected domain in all diagnosis groups, with patients having LAMA2-RD the most severely affected (only $10 \%$ of ambulant patients).

A trend toward a floor effect was seen for the MD_NM-Score D1. The median score in all patients was 3 (range, 2-4); 14 patients (33.5\%) scored 4, indicating severe limitations regarding standing and transfers.

The 3 diagnosis groups showed significant differences for MD_NM-Scores D1 and D3 ( $P=$. 0004 and $P=.02$, respectively) but not for MD_NM-Score and D2 $(P=.13)$. Nonambulant patients scored significantly higher (had more severe conditions) than ambulant patients in all MD_NM-Scores (D1, D2, and D3: $P<.0001, P=.0005$, and $P<.0001$, respectively). 


\section{Concurrent validity of NM-Score}

Table 2 shows the mean values of different assessments used as criterion standards at baseline and the correlation values found between the NM scores and the other measurements. All the correlations were high and significant, except between MD_NMScore D3 and the Jebsen writing subtest $(\rho=.33)$. The Spearman correlation coefficients between NM score D1 and MFM_D1, ACTIVLIM, and hip abduction strength measured by myometry (Myo_Hip_Abd) were the highest and ranged from -.944 to -.811 . The correlations were found to be lower for NM scores D2 and D3.

\section{Informant agreement between MD_NM-Scores and P/C_NM-Scores}

Table 3 lists the distributions of the agreements and disagreements between the levels of classification in each domain. Overall, the patients were fairly evenly distributed among the most impaired levels of each domain, except for NM score D3, for which no patients scored 4. As expected, in all domains, few patients scored 0 , which corresponds to the ability of unaffected subjects. Regarding domain D1, the pairs of raters (P/C, MD) agreed on the level of the NM-Score for 31 patients and disagreed by 1 level for 11; P/Cs scored 9 of 11 patients as more severe (higher score) than MDs estimated. For NM score D1, the informant agreement between P/C_NM-Scores and MD_NM-Scores was excellent $(\kappa=.801 ; 95 \%$ confidence interval [CI], .701-.914).

The disagreements between paired raters were more frequent with NM score D2 (20 disagreements by 1 level and 1 disagreement by 2 levels) and NM score D3 (18 disagreements by 1 level) than with NM score D1, but here we did not find a trend toward overscoring by P/Cs versus MDs (10 vs 11 for D2 and 8 vs 18 for D3) as was the case with NM scores D1. For NM scores D2 and D3, the informant agreement between P/C_NMScores and MD_NM-Scores was considered moderate (NM score D2: $\kappa=.592 ; 95 \%$ CI, . 412-.773; NM score D3: $\kappa=.485$; 95\% CI, .290-.680).

As shown in table 2, the correlation coefficients between the NM scores and most of the criterion standards were not significantly different between P/C_NM-Scores and MD_NMScores, except for MFM D3 and Jebsen_feeding subtest for which significantly higher correlation coefficients were found with the MD_NM-Scores.

\section{Discussion}

The main objective of the current study was to create an English language version of the NM-Score and assess its validity in a population of English-speaking participants with NMD. A secondary objective was to explore the informant agreement between the MD_NM-Scores and P/C_NM-Scores and the differences in agreement according to the disease severity and to the NM-Score domains. ${ }^{13}$

Within the limits of the sample studied here, our results demonstrate that the English version of the NM-Score described accurately the abilities of the participants and was able to classify them reliably into homogeneous groups on the basis of their daily performance whatever their ambulant status or their diagnosis group, or both, thereby providing good evidence for its construct validity. The results confirmed our hypothesis that the NM-Score 
reflects accurately the overall disease severity. The concurrent validity was demonstrated by interesting correlations with the criterion standards. The poor correlations between Jebsen scores and D3 may be explained by the unfavorable effect of a proximal motor function (D2) on positioning and stabilizing the upper limb for adequate distal motor function (D3), especially regarding feeding, writing, or seizing small objects, which are fine motor activities.

Patients and caregivers found the NM-Score easy to complete and provided positive feedback. However, the P/C_NM-Scores tended to be higher than the MD_NM-Scores on D1 (not on D2 or D3). This highlights the usefulness of the NM-Score for patients and families who wish to determine patient performance in daily living activities-for example, for patient self-reported registries. This trend and the level of agreement by domain are similar to previous similar reports. For example, in a study of behavior problems in children with epilepsy, the informant agreement varied according to the domain of the Child Behavior Checklist, and the mothers reported more problems than the teachers. ${ }^{19}$ However, the comparison with the present study is not straightforward because in most cases here, the P/C_NM-Scores were obtained from parents and children together, whereas in the epilepsy example, parents and children responded separately.

The correlations between P/C_NM-Scores and the criterion standards were not significantly higher than those obtained between MD_NM-Scores and these criterion standards. This makes it difficult to determine which score is the most accurate. Since patients/caregivers are the most familiar with their daily performance, they should be carefully interviewed by the clinicians when completing the MD_NM-Score.

Interestingly, the Spearman correlations between MD_NM-Scores and MFM subscores were higher in the present study than in the previous French validation study (.94 vs .88 for D1, . 75 vs . 70 for D2, and .81 vs .49 for D3). It is thus likely that the motor function assessed here (ordinary circumstances, patients with CMD only, and aged 5-19y) is closer to the motor capacity assessed under optimal conditions than in the much more heterogeneous French validation population (various NMDs, ages 6-60y).

Our project is indebted to the work of Palisano et $\mathrm{al}^{20}$ on cerebral palsy, especially regarding the GMFCS. Similar to this instrument, the NM-Score classification was based on a qualitative clinical description of motor function through relevant items or "word pictures." The NM-Score does not rely on performance but rather on patient interview and observation. The raters were asked to carefully read the questionnaire and the instructions before the NM-Score use, but no specific training was provided. However, to avoid incorrect interpretations and overcome differences in clinical experience, we recommend that teams of professionals undergo a training phase, independently score several participants, and then discuss the results before adopting the NM-Score in daily clinical practice. The major difference with the GMFCS is that the NM-Score is expected to change during the course of most NMDs, whereas the GMFCS would remain the same because it was designed to provide a prognosis. 
The English version of the NM-Score involved a multidisciplinary group of experts in the translation and adaptation of the classification. The recourse to a committee for the backtranslations was recommended by several authors to identify misinterpretations and promote expert exchanges. ${ }^{16,21-23}$ Indeed, the back-translation revealed several semantic inconsistencies that were then corrected. For example, the English-speaking cultural approach to disability led to the use of a more positive terminology to describe handicaps compared with the original version (eg, "limitation" instead of "deficiency"). A partial validation study was carried out after translation and adaptation to confirm the metrological properties of the English version, because a complete English cross-cultural translation and adaptation may not have conferred equivalent psychometric properties. ${ }^{24}$

Within the context of the current advances in understanding and treating NMDs, the NMScore should be of interest to practitioners and clinical researchers. In clinical practice, it is an easy-to-use tool to describe patients' performance status in 3 motor function domains. It will ease physician-patient discussions about usual daily living activities at home and prevent focusing on the best patient capacities in the controlled, and limited, environment of a clinic. The scoring system can help health professionals record standardized information on a patient's functional abilities and anticipate any rehabilitative needs. It can also be used to assess the effects of medical interventions, including the use of assistive technology and adaptive equipment, but only when the effects are of sufficient magnitude. In research, the NM-Score is able to describe precisely the motor function of a given population and classify the patients into homogeneous groups of disease severity, for instance, for the purpose of quickly defining trial populations. Unlike the Vignos Scale and Brooke Scale, the NM-Score provides a full description of a patient's motor function in all domains for all NMDs and is therefore superior for patient stratification. However, the NM-Score is as poorly sensitive to change as the Vignos Scale or Brooke Scale ${ }^{25}$; it cannot be used as an outcome measure to evaluate moderate treatment efficacies because the motor function change needed to move from one level to another is too large.

\section{Study limitations}

One study limitation is that all patients had a diagnosis of CMD; however, the range of patient severity was sufficiently broad to test the scale classification. Because of the limited size of the sample and because it consisted only of patients with CMD, though it included walking and nonwalking subjects, the results of the present study cannot be readily generalized without caution. Besides, in the present study setting, it was not possible to analyze the differences between the various assessments (patient, parent/caregiver, both) because of the small sample size; only 2 patients were able to perform self-evaluation.

\section{Conclusions}

The NM-Score provides an accurate functional description of patients with NMDs in terms of motor performance in 3 different functional domains, and enables patient stratification into homogeneous groups. The NM-Score provides a description of the functional status that is not limited to the walking ability; this enables its use over a broad range of neuromuscular disorders. It can be administered by physicians, caregivers, or patients. In population studies, 
instead of simple percentages of specific abilities, the NM-Score provides measurements of disease severity in each of 3 functional domains. If this classification proves as useful as we expect it to be, clinicians will be able to use its objective data for various clinical or research purposes.

\section{Acknowledgments}

Supported by the National Institute of Neurological Disorders and Stroke, the French Association Against Myopathies (AFM), the French Society of Physical Medicine and Rehabilitation (SOFMER), the French Speaking Society for Study and Research in Children with Disabilities (SFERHE), the Hospices Civils de Lyon, the Philippe Foundation, and Bouillat Terrier and Deage.

We thank Pierre Bigot, MD, for his revisions of the manuscript and assistance with the references section, Philippe Meilleur for his contribution of translating the NM-Score into English, and Joan Austin, PhD, for her revisions of the manuscript. We gratefully acknowledge the contributions of the experts who produced the initial French version of NM-Score: M.C. d'Anjou, MD (Saint-Étienne, France), F. Aubert, PT (Lyon, France), I. Badoil, OT (Lyon, France), G. Bassez, MD (Créteil, France), A. Barrière, OT (Lyon, France), B. Berger, MD (Grenoble, France), A. Berruyer, PT (Lyon, France), F. Bouhour, MD (Lyon, France), Y. Chartier, PT (Lyon, France), J.M. Cuisset, MD (Lille, France), M.C. Delmas, MD (Saint Denis de la Réunion, France), D. Denis, PT (Lyon, France), C. Fafin, PT (Nice, France), L. Féasson, MD (Saint-Etienne, France), S. Fontaine, MD (Lyon, France), M. Fournier-Mehouas, MD (Nice, France), V. Germa, OT (Paris, France), F. Girardot, PT (Lyon, France), N. Goemans, PT (Leuven, Belgium), H. Hovart, PT (Lille, France), V. Humbertclaude, MD (Montpellier, France), V. Kinet, PT (Bruxelles, Belgium), D. Laridant, OT (Bruxelles, Belgium), C. de Lattre, MD (Lyon, France), A. Le Flem, OT (Lyon, France), E. Luc-Pupat, OT (Lyon, France), V. Manel, MD (Lyon, France), F. Margirier, PT (Lyon, France), C. Payet, PT (Montpellier, France), S. Quijano-Roy (Garches, France), N. Pellegrini, MD (Garches, France), I. Penisson-Besnier, MD (Angers, France), A. Renders, MD (Bruxelles, Belgium), G. Rode, MD (Lyon, France), A.P. Rouyer, OT (Lyon, France), F. Rumeau, MD (Vandoeuvre-les-Nancy, France), D. Salicio, PT (Hendaye, France), V. Spehrs, MD (Lausanne, Switzerland), V. Tanant, PT (Nice, France), V. Tiffreau, MD (Lille, France), A, Urtizberea, MD (Hendaye, France), J.P. Vadot, MD (Lyon, France), E. Viehweger, MD (Marseille, France), and D. Vincent-Genod, PT (Lyon, France).

\section{List of abbreviations}

ACTIVLIM activity limitations for patients with upper and/or lower limb impairments

CI confidence interval

CMD congenital muscular dystrophy

GMFCS Gross Motor Function Classification System

LAMA2-RDlaminin alpha 2-related disease

MD medical doctor

MFM Motor Function Measure

NIH National Institutes of Health

NM neuromuscular

NMD neuromuscular disease

P/C patient/caregiver 


\section{References}

1. Rossi, R.; Alexander, M.; Cuccurullo, S. Pediatric rehabilitation. In: Cuccurullo, SJ., editor. Physical medicine and rehabilitation board review. New York: Demos Medical; 2004. p. 713-808.

2. Iannaccone ST. Outcome measures for pediatric spinal muscular atrophy. Arch Neurol. 2002; 59:1445-1450. [PubMed: 12223032]

3. Scott E, Mawson SJ. Measurement in Duchenne muscular dystrophy: considerations in the development of a neuromuscular assessment tool. Dev Med Child Neurol. 2006; 48:540-544. [PubMed: 16700951]

4. O'Hagen JM, Glanzman AM, McDermott MP, et al. An expanded version of the Hammersmith Functional Motor Scale for SMA II and III patients. Neuromuscul Disord. 2007; 17:693-697. [PubMed: 17658255]

5. Scott OM, Hyde SA, Goddard C, Dubowitz V. Quantitation of muscle function in children: a prospective study in Duchenne muscular dystrophy. Muscle Nerve. 1982; 5:291-301. [PubMed: 7099196]

6. Krosschell KJ, Maczulski JA, Crawford TO, Scott C, Swoboda KJ. A modified Hammersmith Functional Motor Scale for use in multicenter research on spinal muscular atrophy. Neuromuscul Disord. 2006; 16:417-426. [PubMed: 16750368]

7. Mazzone E, Martinelli D, Berardinelli A, et al. North Star Ambulatory Assessment, 6-minute walk test and timed items in ambulant boys with Duchenne muscular dystrophy. Neuromuscul Disord. 2010; 20:712-716. [PubMed: 20634072]

8. Berard C, Payan C, Hodgkinson I, Fermanian J. A motor function measure for neuromuscular diseases. Construction and validation study. Neuromuscul Disord. 2005; 15:463-470. [PubMed: 16106528]

9. Palisano R, Rosenbaum P, Walter S, Russell D, Wood E, Galuppi B. Development and reliability of a system to classify gross motor function in children with cerebral palsy. Dev Med Child Neurol. 1997; 39:214-223. [PubMed: 9183258]

10. Brooke MH, Griggs RC, Mendell JR, Fenichel GM, Shumate JB, Pellegrino RJ. Clinical trial in Duchenne dystrophy. I. The design of the protocol. Muscle Nerve. 1981; 4:186-197. [PubMed: 7017401]

11. Brooke MH, Fenichel GM, Griggs RC, et al. Clinical investigation in Duchenne dystrophy: 2. Determination of the "power" of therapeutic trials based on the natural history. Muscle Nerve. 1983; 6:91-103. [PubMed: 6343858]

12. Vignos PJ Jr, Spencer GE Jr, Archibald KC. Management of progressive muscular dystrophy in childhood. JAMA. 1963; 184:89-96. [PubMed: 13997180]

13. Vuillerot $\mathrm{C}$, Rippert $\mathrm{P}$, Roche $\mathrm{S}$, et al. Development and validation of a motor function classification in patients with neuromuscular disease: the NM-Score. Ann Phys Rehabil Med. 2013; 56:673-686. [PubMed: 24231198]

14. Vandervelde L, Van den Bergh PY, Goemans N, Thonnard JL. ACTIVLIM: a Rasch-built measure of activity limitations in children and adults with neuromuscular disorders. Neuromuscul Disord. 2007; 17:459-469. [PubMed: 17433675]

15. Davis Sears E, Chung KC. Validity and responsiveness of the Jebsen-Taylor Hand Function Test. J Hand Surg [Am]. 2010; 35:30-37.

16. Guillemin F, Bombardier C, Beaton D. Cross-cultural adaptation of health-related quality of life measures: literature review and proposed guidelines. J Clin Epidemiol. 1993; 46:1417-1432. [PubMed: 8263569]

17. Beenakker EA, van der Hoeven JH, Fock JM, Maurits NM. Reference values of maximum isometric muscle force obtained in 270 children aged 4-16 years by hand-held dynamometry. Neuromuscul Disord. 2001; 11:441-446. [PubMed: 11404114]

18. Colton, T. Statistics in medicine. Boston: Little, Brown and Co; 1974. p. 372

19. Huberty TJ, Austin JK, Harezlak J, Dunn DW, Ambrosius WT. Informant agreement in behavior ratings for children with epilepsy. Epilepsy Behav. 2000; 1:427-435. [PubMed: 12737832]

20. Palisano RJ, Cameron D, Rosenbaum PL, Walter SD, Russell D. Stability of the Gross Motor Function Classification System. Dev Med Child Neurol. 2006; 48:424-428. [PubMed: 16700931] 
21. Geisinger, K. Testing and assessment in cross-cultural psychology. In: Graham, JR.; Naglieri, JA., editors. Handbook of psychology. New York: John Wiley \& Sons; 2003. p. 95-117.

22. Van der Vijver, F.; Leung, K. Methods and data analysis for cross-cultural research. Thousand Oaks: Sage; 1997.

23. Flaherty JA, Gaviria FM, Pathak D, et al. Developing instruments for cross-cultural psychiatric research. J Nerv Ment Dis. 1988; 176:257-263. [PubMed: 3367140]

24. Vallerand R. Toward a methodology for the transcultural validation of psychological questionnaires: implications for research in the French language [French]. Can Psychol. 1989; 30:662-680.

25. Lue YJ, Su CY, Yang RC, et al. Development and validation of a muscular dystrophy-specific functional rating scale. Clin Rehabil. 2006; 20:804-817. [PubMed: 17005504]

\section{Appendix 1}

The NM-Score classification.

\begin{tabular}{|c|c|}
\hline \multicolumn{2}{|c|}{ NM-Score D1: Standing position and transfers } \\
\hline$\square \mathbf{0}$ & No impairment: Walking ability, running and jumping are comparable to their peers. \\
\hline$\square \mathbf{1}$ & $\begin{array}{l}\text { Mild impairment: The individuals can walk (walking distance }>500 \mathrm{~m} \text { ) in town (sidewalks), } \\
\text { in supermarkets and on uneven ground. Many individuals may run and jump but with reduced } \\
\text { speed, impaired balance and/or compensatory strategies. }\end{array}$ \\
\hline$\square 2$ & $\begin{array}{l}\text { Moderate impairment: The individuals can walk over limited distances in the } \\
\text { neighborhood, within school or a workplace (walking distance }<500 \mathrm{~m} \text { ). Very few } \\
\text { individuals have the ability to run and jump. }\end{array}$ \\
\hline$\square 3$ & $\begin{array}{l}\text { Severe impairment: Upright mobility is very limited. Some individuals are able to take a few } \\
\text { steps inside the home with an assistive device or another person to assist, but upright mobility } \\
\text { is not the primary method of mobility. }\end{array}$ \\
\hline$\square 4$ & $\begin{array}{l}\text { Very severe impairment: Individuals cannot take any steps or stand up. No transfer of } \\
\text { weight to the feet can be achieved to move from seated to standing position. }\end{array}$ \\
\hline \multicolumn{2}{|c|}{ NM-Score D2: Axial and proximal motor function } \\
\hline$\square 0$ & $\begin{array}{l}\text { No impairment: Seated ability, proximal motor function of limbs, and head control are } \\
\text { comparable to their peers. }\end{array}$ \\
\hline$\square \mathbf{1}$ & $\begin{array}{l}\text { Mild impairment: Individuals can stay in an upright, seated position for an unlimited } \\
\text { amount of time (all day at school or at work) on a regular seat. Holding a seated position may } \\
\text { be qualitatively abnormal due to postural kyphosis or a level of fatigue requiring the support } \\
\text { of an upper limb or a chair back. Head control is normal for an unlimited amount of time. }\end{array}$ \\
\hline$\square 2$ & $\begin{array}{l}\text { Moderate impairment: Individuals can sit without support but for a limited amount of time. } \\
\text { Individuals able to sit for unlimited amount of time will be classified in this level of function } \\
\text { if: } \\
\rightarrow \text { the individual is unable to lift or carry objects above shoulder level and/or has difficulty } \\
\text { carrying and handling of heavy objects and/or } \\
\rightarrow \text { head control is compromised or only possible for short periods of time. }\end{array}$ \\
\hline$\square 3$ & $\begin{array}{l}\text { Severe impairment: The seated position without support is limited to a few important and } \\
\text { purely functional situations (dressing, positioning, donning/doffing a body jacket, sitting on } \\
\text { the toilet). }\end{array}$ \\
\hline$\square 4$ & $\begin{array}{l}\text { Very severe impairment: Individuals cannot sit up unassisted. The seated position is only } \\
\text { possible with the use of adaptive equipment. }\end{array}$ \\
\hline \multicolumn{2}{|c|}{ NM-Score D3: Distal motor function } \\
\hline$\square 0$ & No impairment: Object manipulation and distal motor function are comparable to their peers. \\
\hline$\square \mathbf{1}$ & $\begin{array}{l}\text { Mild impairment: The majority of object manipulation and distal motor function in daily life } \\
\text { activities is preserved. Manual activities requiring greater strength are possible but with } \\
\text { difficulty, affecting speed, fatigue levels and the need for compensatory movements. } \\
\text { Intention tremors or a lack of precision can be the only impairment observed during the } \\
\text { activity. }\end{array}$ \\
\hline
\end{tabular}


\begin{tabular}{|l|l}
$\square$ & Moderate impairment: Object manipulation and distal motor function are limited due to a
\end{tabular} lack of strength and/or poor control of movement. The individuals cannot perform certain manual activities requiring strength and significant dexterity. Easier manual activities are possible, fully performed but with difficulty, slowness, fatigue or need to compensate. The objects may be modified in order to facilitate their use (large pencil, adapted lids or covers).

$\square 3$ Severe impairment: Individuals cannot perform manual activities requiring strength and significant dexterity. Object manipulation and distal motor function during easy manual activity may be partially accomplished. In order for these activities to be carried out in full, adaptive equipment or assistance from another person is required.

$\square 4$ Very severe impairment: The great majority of object manipulation and distal motor function in daily life activities is very difficult or unable to be accomplished. 


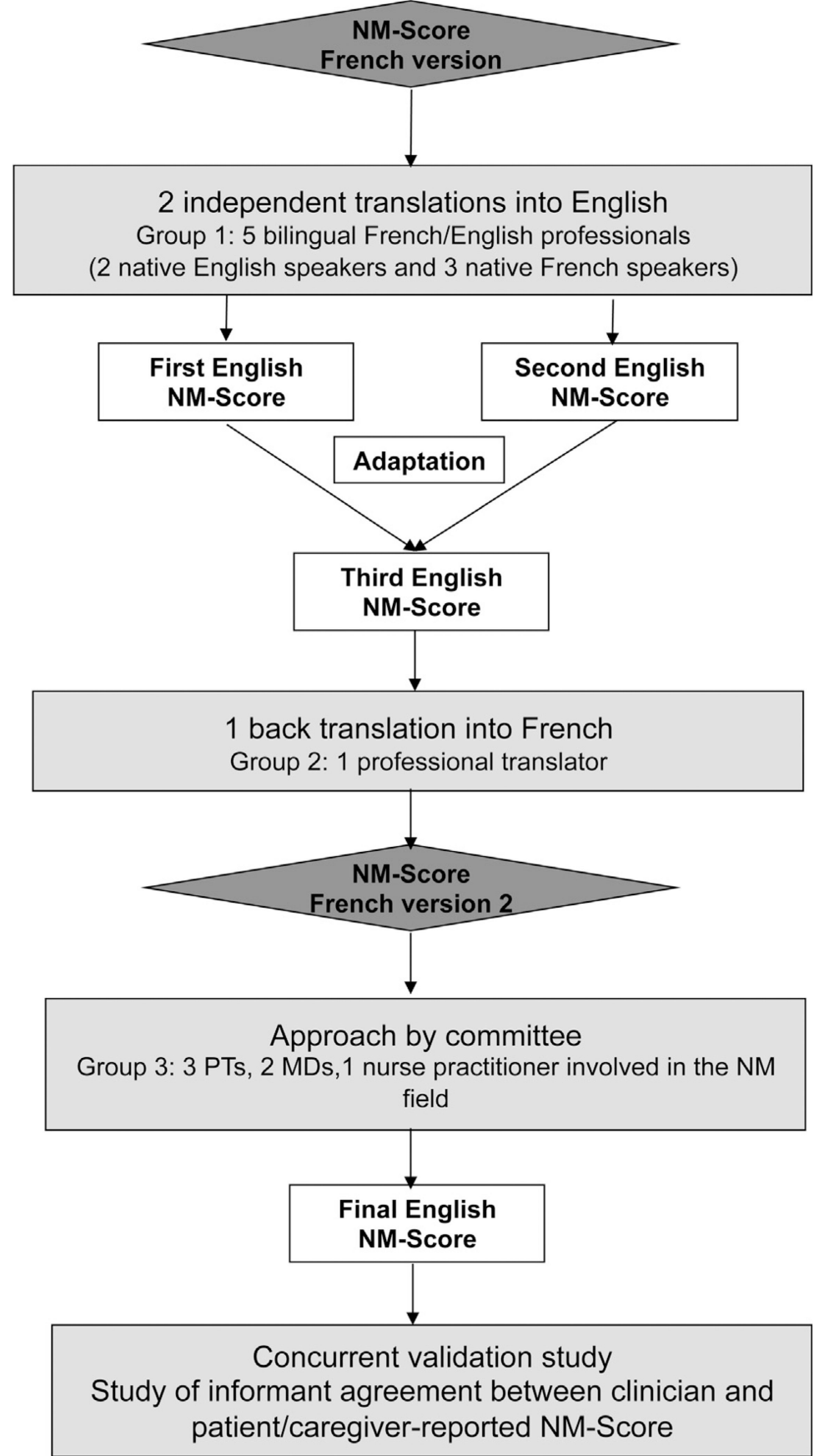

Fig 1.

The English NM-Score validation process. Abbreviation: PT, physical therapist. 


\section{Table 1}

Patients' characteristics

\begin{tabular}{|llll|}
\hline & $\begin{array}{l}\text { Laminin a2- } \\
\text { Deficient } \\
\text { CMD (naracteristics }\end{array}$ & $\begin{array}{l}\text { Collagen } \\
\text { 6-Deficient } \\
\text { CMD (n = 20) }\end{array}$ & $\begin{array}{l}\text { Undiagnosed } \\
\text { CMD (n= 4) }\end{array}$ \\
\hline Age (y) & $9.3 \pm 2.4$ & $12.7 \pm 3.8$ & $8.5 \pm 0.5$ \\
Boys (\%) & 40 & 60 & 75 \\
Ambulant (\%) & 10 & 55 & 100 \\
NM score by MD & & & \\
MD_NM-Score D1 & $3(2-4)$ & $2(1-4)$ & $1(0-2)$ \\
MD_NM-Score D2 & $2(1-4)$ & $1.5(0-4)$ & $1(0-1)$ \\
MD_NM-Score D3 & $2(0-3)$ & $1.5(0-2)$ & $1.5(0-3)$ \\
NM score by P/C & & & $1(0-2)$ \\
P/C_NM-Score D1 & $4(1-4)$ & $3(1-4)$ & $1.5(0-2)$ \\
P/C_NM-Score D2 & $2(1-4)$ & $1.5(0-4)$ & $1.5(0-2)$ \\
P/C_NM-Score D3 & $2(1-3)$ & $1.5(0-2)$ & \\
\hline
\end{tabular}

NOTE. Values are mean $\pm \mathrm{SD}$, median (range), or as otherwise indicated.

Abbreviations: D1, domain 1 (standing position and transfers); D2, domain 2 (axial and proximal motor function); D3, domain 3 (distal motor function). 
Table 2

Spearman correlation coefficients between criterion standards and NM-Scores (MD_NM-Scores and P/ C_NM-Scores)

\begin{tabular}{|c|c|c|c|c|}
\hline \multirow{2}{*}{$\begin{array}{l}\text { Variables Used for } \\
\text { Concurrent Validity }\end{array}$} & \multirow[b]{2}{*}{ Values } & \multirow{2}{*}{$\begin{array}{l}\text { NM-Score } \\
\text { Tested }\end{array}$} & \multicolumn{2}{|c|}{ Spearman $\rho$} \\
\hline & & & MD & $\mathbf{P} / \mathbf{C}$ \\
\hline MFM_D1 (\%) & $24.0 \pm 26.6$ & D1 & $-.94^{*}$ & $-.94^{*}$ \\
\hline MFM_D2 (\%) & $65.8 \pm 30.0$ & $\mathrm{D} 2$ & $-.75^{*}$ & $-.62 *$ \\
\hline MFM_D3 (\%) & $81.0 \pm 22.1$ & D3 & $-.81^{*}$ & $-.61^{*}$ \\
\hline \multirow[t]{3}{*}{ ACTIVLIM (logit) } & $-1.13 \pm 2.9$ & D1 & $-.89^{*}$ & $-.90^{*}$ \\
\hline & NA & $\mathrm{D} 2$ & $-.70 *$ & $-.77^{*}$ \\
\hline & NA & D3 & $-.72 *$ & $-.66^{*}$ \\
\hline Myo_Hip_Abd (\% NV) & $18.6 \pm 19.4$ & D1 & $-.81^{*}$ & $-.76^{*}$ \\
\hline Myo_Knee_Ext (\% NV) & $19.5 \pm 12.3$ & D1 & $-.72 *$ & $-.69^{*}$ \\
\hline Myo_Elbow_Ext (\% NV) & $16.1 \pm 11$ & D2 & $-.62 *$ & $-.58^{*}$ \\
\hline Brooke Upper Extremity & $1(1-5)$ & D2 & $.72 *$ & NA \\
\hline Scale & & D3 & $.70^{*}$ & NA \\
\hline Jebsen_writing (s) & $45.6 \pm 49.3$ & D3 & .33 & .29 \\
\hline Jebsen_cards (s) & $19.3 \pm 29.4$ & D3 & $.66^{*}$ & $.51^{\dagger}$ \\
\hline Jebsen_small objects (s) & $24.2 \pm 30.6$ & D3 & $.57^{\dagger}$ & $.54^{\dagger}$ \\
\hline Jebsen_feeding (s) & $27.4 \pm 30.9$ & D3 & $.61^{*}$ & .32 \\
\hline Jebsen_checkers (s) & $9.9 \pm 12.7$ & D3 & $.69^{*}$ & $.64^{*}$ \\
\hline Jebsen_light objects (s) & $27.9 \pm 47.7$ & D3 & $.61^{\dagger}$ & $.62^{*}$ \\
\hline Jebsen_heavy objects (s) & $48.8 \pm 61.0$ & D3 & $.71^{*}$ & $.66^{*}$ \\
\hline
\end{tabular}

NOTE. Values are mean \pm SD for quantitative variables, median (range) for categorical variables, or as otherwise indicated.

Abbreviations: D1, domain 1 (standing position and transfers); D2, domain 2 (axial and proximal motor function); D3, domain 3 (distal motor function); Myo_Elbow_Ext, myometry data for elbow extension; Myo-Hip-Abd, myometry data for hip abduction; Myo_Knee_Ext, myometry data for knee extension; NA, not applicable; NV, normal value.

* $P<.001$.

${ }^{\dagger} P<.01$. 


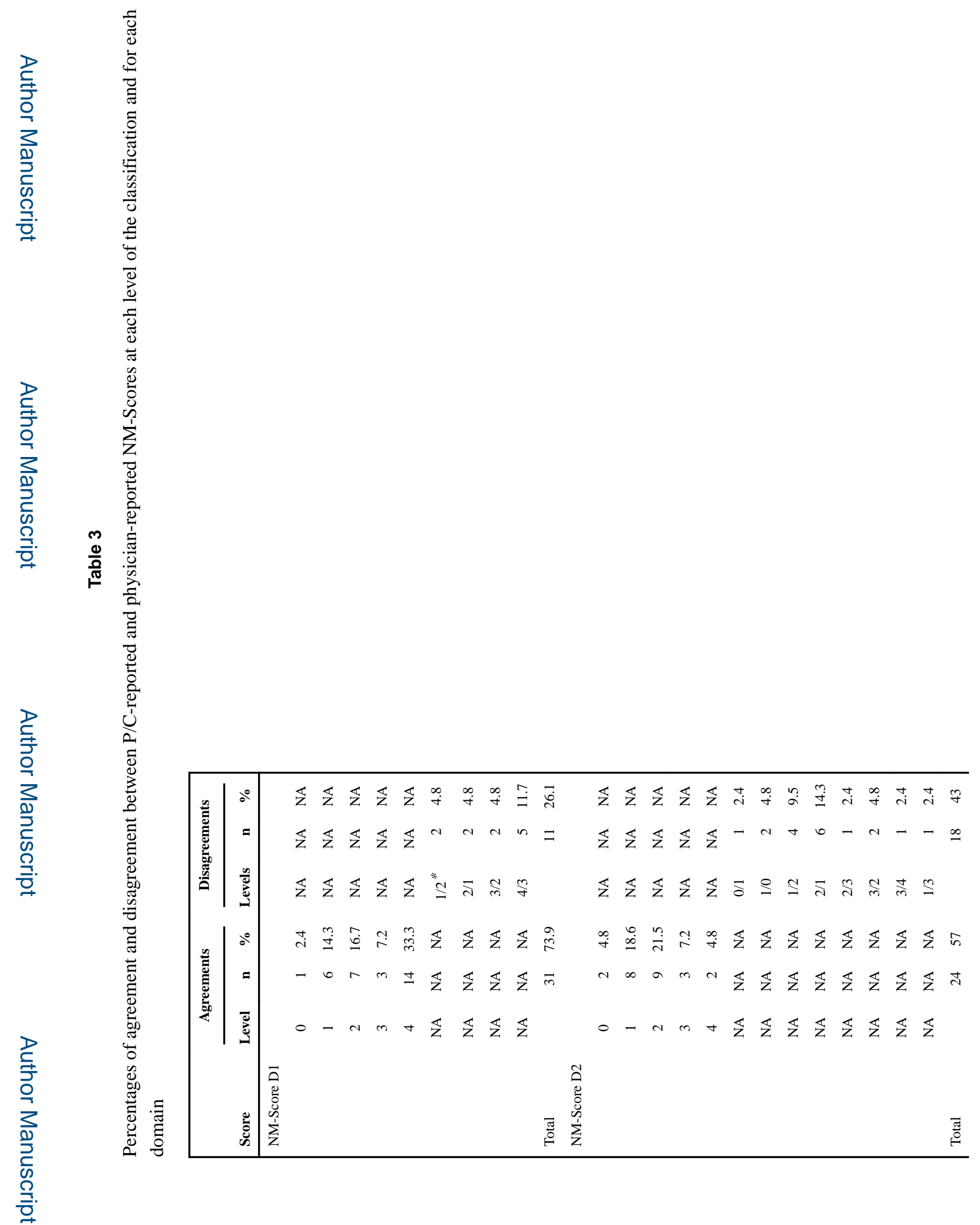




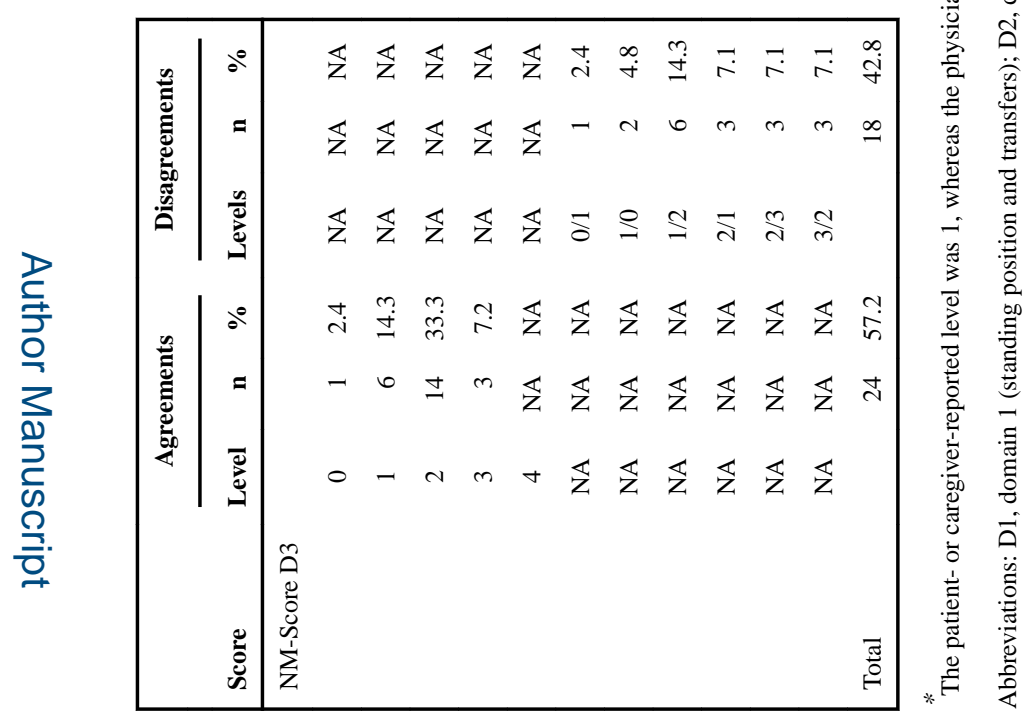

로을 